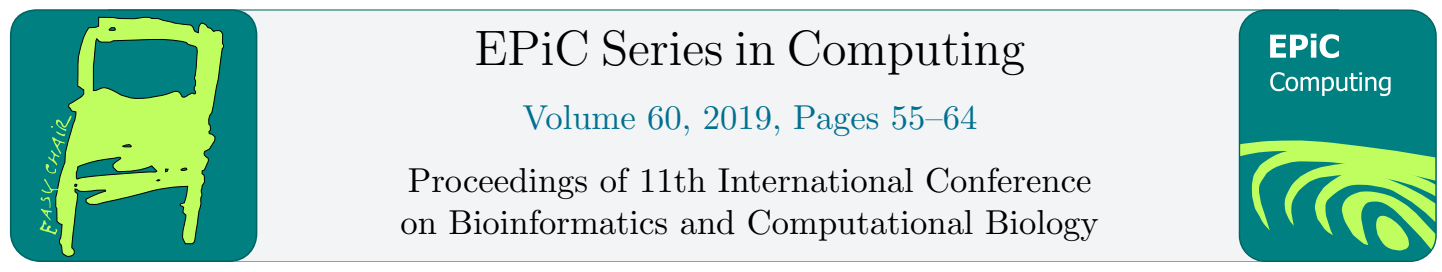

\title{
Comparison of Type I Interferon Expression in Adult and Neonatal Mice during Respiratory Viral Infection
}

\author{
Zifeng Liang ${ }^{1}$ \\ ${ }^{1}$ Miss Hall's School \\ zliang@misshalls.org
}

\begin{abstract}
The aim of this paper is to identify the difference of type I interferon expression in 2day neonatal and six-to-eight-weeks adult mice infected by Sendai virus ( $\mathrm{SeV}$ ), a singlestranded RNA virus of the family Paramyxoviridae. Sendai virus mimics the influence of respiratory syncytial virus (RSV) on humans, but does not infect humans. Although RSV has a fatal impact on people across age groups, little is understood about this common virus and the disparity between neonatal and adult immune response to it. It has been suggested by past findings that Type I interferon mRNA is present in higher levels in adults than in neonates, however there is a greater amount of interferon proteins in neonates rather than adults. To test the hypothesis that neonates are more capable of interferon production and preventing the translation of viral protein, I observed mouse models of respiratory viral infection and determined the expression of IFN- $\alpha 1$, IFN- $\alpha 2$, IFN- $\alpha 5$, IFN- $\alpha 6$, IFN- $\alpha 7$, IFN- $\beta$ in archived mouse lung tissue samples harvested on different days post-infection with quantitative real time PCR. Expression of Glyceraldehyde 3-phosphate dehydrogenase(GAPDH), a housekeeping gene expressed constitutively in all mouse models, was used as a positive control of the experiment. To determine the ideal concentration of primer used in qPCR, primer reconstitution, primer optimization, and gel electrophoresis were conducted in advance. In addition, technical replicates and biological replicates were used to reduce error and confirm results in qPCR. In accordance with previous discovery, I found an upward trend in adults' interferon expression from post-infection day 1 to day 5 , and levels off in day 7 . In contrast, neonatal levels were much higher on day 1 and remained high over the course of infection. This explains how type I interferon expression is altered in neonates to help them clear the virus at the same efficiency as adults without causing inflammation. Future research on immune response differences in human infection should focus on the evaluation of interferon protein amounts, as well as the analysis of activation of molecules downstream of the type I interferon receptors, such as signal transducer and activator of transcription (STAT) protein family. It is also crucial to compare immune cells like macrophages and natural killer cell activity in adult and neonatal mice during viral infection.
\end{abstract}




\section{Introduction}

\section{Respiratory viral infection}

Paramyxoviruses are a fa family of enveloped viruses with linear, negative-sense, and singlestranded RNA. The paramyxoviridae family includes respiratory syncytial virus (RSV), the major cause of bronchiolitis and pneumonia in newborns and young children, as well as human parainfluenza virus (HPIV), the second leading cause of respiratory disease. Paramyxoviruses are responsible for $20 \%$ of childhood death worldwide. (Bhattacharya) Additionally, respiratory viruses are detected in $90 \%$ of hospitalized children. It is transmitted through air particles as we breathe. Once the virus gets into respiratory epithelial cells, the viral RNA strand is converted into a complementary positive-sense strand and subsequently translated. The virus exits the host cell by budding. As a result, infected area could expand exponentially in a very short interval upon infection.

Symptoms of RSV infection vary from simple cold to bronchitis, inflammation in small airways in the lungs as immune cells' attempt to kill invading pathogens. This inflammation, however, may cause serious pulmonary injury. It affects people across many age groups, especially infants less than 12 months of age and older adults. In serious conditions, this infection can be deadly. So far, there has not been any functional vaccines or proven antiviral therapeutic treatment. (Bhattacharya)

\section{Type I Interferons}

Following infection with a paramyxovirus, cells in the lungs begin to produce interferons (IFNs). Interferons are a group of cytokines that interfere with viral replication in innate immune response. They are released by the infected cell and travel to neighboring cells where they bind to cellular receptors to initiate an anti-viral response. The interferons are upregulated when an infected cell detects pathogen-associated molecular patterns (PAMPs) such as viral nucleic acids. PAMPs bind to pattern recognition receptors (PRRs), which initiate a signal transduction cascade culminating in the transcription of the type I IFN genes. (McNab)

Mammalian interferons are classified into three types: Type I interferon, Type II interferon, and Type III interferon. Type I interferon includes the two major kinds IFN- $\alpha$ and IFN- $\beta$, as well as

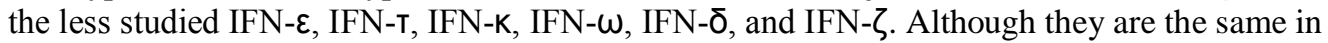
structure and function, IFN- $\alpha$ is secreted by virally infected leukocytes like macrophages, monocytes, and lymphocytes, and IFN- $\beta$ is secreted by fibroblasts and epithelial cells. Type I interferons alert immune cells like natural killer cells and macrophages that infection has occurred. Viral nucleic acid is then degraded and viral protein synthesis is inhibited. Type II interferon, IFN- $\gamma$, is produced by activated T lymphocytes and natural killer cells. They stimulate the activity of macrophages in adaptive immunity. Type III interferons, IFN- $\gamma$, signal through a distinct receptor complex to induce immune response. (Owen)

During infection with an RNA virus, the type I interferons are produced by activation of the RIG-I-MAVS signal pathway. In this pathway, the RIG-I-like receptors (RLR), including Retinoic acid-inducible gene I (RIG-I), Melanoma differentiation-associated gene 5 (MDA5), and Laboratory of genetics and physiology 2 (LGP2), bind to and activate TANK-binding-Kinase 1 (NBK1). Active NBK1 then promotes the phosphorylation of interferon transcription factors IRF-3 and IRF-7, which leads to the production of IFN- $\alpha$ and IFN- $\beta$ proteins. Upon their release from an infected cell, they bind to the interferon- $\alpha / \beta$ receptor 1 . This receptor has two subunits: the (IFNAR 1 ) subunit associated with Janus Kinase 1(JAK1) and the IFNAR 2 subunit associated with Tyrosine kinase 2(Tyk2). Signaling through the IFNAR pathway initiates an anti-viral response in the infected cell. This pathway begins with the recruitment and activation of JAK1 and Tyk2, which then activate specific Signal Transducer and Activator of Transcription(STAT). STAT1/1 and STAT1/2 induce the expression of various viral-replication-blocking genes such as protein kinase $\mathrm{R}(\mathrm{PKR})$ and $\mathrm{Mx}$ proteins. This type I IFN mediated anti-viral response is very important for keeping viral levels in check when the adaptive response is activated. (Noton). 


\section{Neonatal response to respiratory viral infection}

It is commonly believed that infants constitute the most vulnerable group to serious respiratory viral infection due to their underdeveloped immune defense system and narrower airways, but surprisingly, previous studies using a mouse model of paramyxoviral infection demonstrated that neonates had significantly reduced inflammation in the lung compared to adult mice. To date, few studies have been done to understand the cause that leads to the difference in neonatal and adult response to this respiratory viral infection. (Decker)

This disparity was evidenced by reduced levels of the cytokines TNF-a and IFN- $\alpha$ and the chemokine CXCL2. The neonates were able to clear the virus with a similar efficiency, and they had similar numbers of virus-specific CD8+ cytotoxic T lymphocytes (Bhattacharya, Virology Journal). Because inflammation was reduced, it is important to understand how virus replication was kept in check during the early days of infection in neonates. Preliminary studies showed that IFN- $\alpha$ mRNA expression in whole lung was significantly reduced in neonates compared to adults after day 1 (Figure 1A). In comparison, IFN- $\beta$ protein expression was increased in neonates compared to adults (Figure 1B). This suggested that there is a unique post-transcriptional regulation employed only by the neonates.

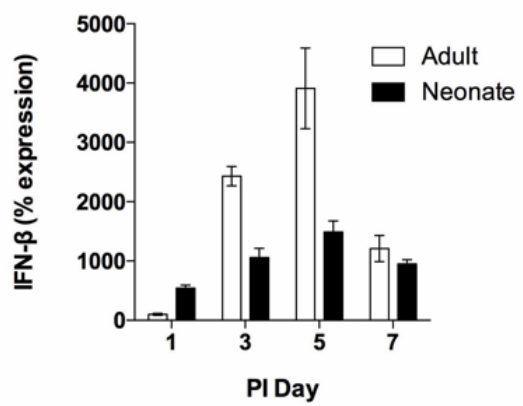

(A)

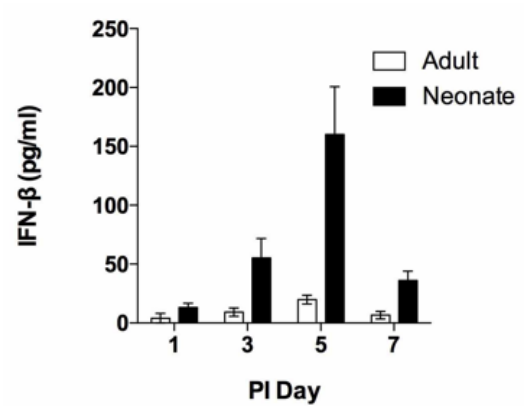

(B)

Figure 1. Adult (6-8 week old) and neonatal (2-day old) C57BL/6 mice were infected with $\mathrm{SeV}$ (500 pfu/g body weight). Lungs were harvested at the indicated times and IFN $-\beta$ mRNA expression was determined by real-time PCR (A). Protein levels were determined by ELISA (B). $p<0.05$. (The Shornick Laboratory)

\section{Hypothesis}

IFN- $\alpha$ gene expression will be upregulated immediately after infection then decrease to normal levels as the infection is gradually cleared by the immune function in both age classes. In neonates, I expect IFN- $\alpha$ to be present in higher amounts than adults so that it is explained why the infection could be cleared equally efficiently but with less inflammation than adults. Our approach is to analyze IFN expression in infected adult and neonatal mice lungs by performing qPCR. Realtime PCR quantifies the levels of gene expression with cycle threshold (CT) value, and is therefore the optimal method to assess the amount of DNA molecules present in a sample. 


\section{Methods}

This project was approved by Saint Louis University Institutional Biological \& Chemical (IBC) Safety Committee Protocol (ID: 2016-24547) on June 4, 2018.

\section{Mouse Model}

The experiment was performed according to St. Louis University Institutional Animal Care and Use Committee protocol \#1586. Ten wild type adult mice and ten 2-day neonatal mice in the strain C57BL/6 were infected with 500 pfu/g body weight Sendai Virus (American Type Culture Collection, Manassas, VA). Adult mice ranged from 6-week old to 8-week old. All mice were held in pathogen-free, sterile cages. Prior to infection, adult mice were anesthetized with ketamine and xylazine. The virus was administered in different volume of Phosphate Buffered Saline in adult and neonate in correspondence to their various weights. Virus in $30 \mathrm{uL}$ of PBS was inoculated intranasally in adult mice, and 6uL for the neonate without anesthesia. Weights were taken daily for one-week post-infection to observe the influence of infection on weight loss.

Both adult mice and neonates were euthanized with carbon dioxide on post-infection day 0 , day 1 , day 3 , day 5 , and day 7 . Lung tissues were harvested and stored in $-80^{\circ} \mathrm{C}$. Tissues were snap frozen in a liquid nitrogen bath, and homogenized with mortar and pestle into a layer of very fine dust. RNA was extracted from the lung tissue using the RNAspin Mini RNA Extraction Kit (GE Healthcare) after lysis, filtration, RNA binding, desalting, DNA digestion, wash and dry, and elusion.

\section{Primer Reconstitution}

Lyophilized primers were purchased (Integrated DNA Technologies), $100 \mu \mathrm{M}$ concentration stock solutions were prepared with nuclease free water. With the stock solution, serial dilution was performed to obtain three different concentrations of primers: $10.0 \mu \mathrm{M}, 5.0 \mu \mathrm{M}, 2.5 \mu \mathrm{M}$. Six primer sets, twelve primers in total, were reconstituted: IFN $\alpha$ 1: forward: 5'-GAG AAG AAA CAC AGC CCC TG-3'; reverse: 5'-TCA GTC TTC CCA GCA CAT TG-3'. IFNa2: forward: 5'-GTG CAG GAA CCT CTG AC-3'; reverse: 5'-CTT CTG CTC TGA CCA CCT CC-3'. IFN $\alpha$ 5: forward: 5'-GAC TCA TCT GCT GCA TGG AA-3'; reverse: 5'-TGT TGC ATC ACA CAG GCT TT-3'. IFN $\alpha 6$ : forward: 5' -TCC ATC AGC AGC TCA ATG AC-3'; reverse: 5'-TAT GTC CTC ACA GCC AGC AG-3'. IFN 27 : forward: 5'-CTG CCT GAA GGA CAG AAA GG-3'; reverse: 5'-ATG CAG AAC ACA GAG GGC TT-3'. IFN $\beta$ : forward: 5'-TCT GGA GCA TCT CTT GGA TGG CAA-3'; reverse: 5'-TCC AGC TCC

\begin{tabular}{|c|c|c|}
\hline Day PI & Adult & Neonate \\
\hline \multirow{2}{*}{0} & 28001 & F1 \\
\cline { 2 - 3 } & 28002 & F2 \\
\hline \multirow{2}{*}{1} & 28004 & F6 \\
\cline { 2 - 3 } & 28005 & F7 \\
\hline \multirow{2}{*}{3} & 28009 & F10 \\
\cline { 2 - 3 } & 28010 & F11 \\
\hline \multirow{2}{*}{5} & 28011 & F14 \\
\cline { 2 - 3 } & 28012 & F15 \\
\hline 7 & 28013 & F18 \\
\cline { 2 - 3 } & 28014 & F19 \\
\hline
\end{tabular}
AAG AAA GGA CGA ACA-3'.

Table 1. List of adult and neonatal mouse lung samples. Two biological replicates of each sample were used to reduce error.

\section{Primer Optimization}

Primers were optimized in a 96-Well 0.1-mL Block by QuantStudio ${ }^{\circledR} 5$ System. Each well contained 14 $\mathrm{uL}$ of master mix, which consisted of $14 \mathrm{uL}$ of PerfeCTa SYBR $®$ green Supermix and $4 \mathrm{uL}$ of nuclease free water. A forward primer was then paired with its reverse primer. 4 $\mathrm{uL}$ of each of the two diluted primer were pipetted into the corresponding well as well as $2 \mathrm{uL}$ of infected mice cDNA (diluted 1:5). In order to confirm results, a technical replicate for each sample and a negative control without cDNA were also used. 
Primer Optimization PCR was carried out on a continuous cycle made up of three stages: Hold Stage, PCR Stage, and Melt Curve Stage. In Hold Stage, plates were run at $94^{\circ} \mathrm{C}$ for two minutes. In the second stage, they were cycled 30 times at $94^{\circ} \mathrm{C}$ for 30 seconds, $60^{\circ} \mathrm{C}$ for 30 seconds, and $72^{\circ} \mathrm{C}$ for one minute. At last, melt curve temperatures were set to be $95^{\circ} \mathrm{C}$ for 15 seconds, $60^{\circ} \mathrm{C}$ for one minute, and $95^{\circ} \mathrm{C}$ for 15 seconds.

Melt curves for each sample were evaluated to determine the concentration that displayed the highest peak, indicating the most abundant amplification. Those that contained extra bumps or multiple peaks in their melt curves were discarded.

\section{Agarose Gel Electrophoresis}

Agarose gel electrophoresis of the PCR products was performed to determine product size and specificity. Agarose powder was mixed with 1x Tris/Borate/EDTA buffer solution (1X TBE) to make $1 \%$ agarose solution. After the solution solidified in the gel electrophoresis apparatus, $4 \mathrm{uL}$ of $6 \mathrm{x}$ loading dye and $6 \mathrm{uL}$ of each primer was loaded into wells, except that the first well was loaded with $12 \mathrm{uL}$ of DNA ladder as a standard for measuring the size of bands in subsequent sample wells. Each well contained an interferon primer of varying concentration and one well contained $12 \mathrm{uL}$ of DNA ladder for fragment length determination. The gel was immersed in 1x TBE running buffer and run in Horizon 58 box connected to a BRL Technologies machine at 160 volts. After 60 minutes, the gel was viewed in a UV light box. One single band at size $91 \mathrm{bp}$ is expected to be seen in IFN- $\alpha 1$ sample, $127 \mathrm{bp}$ in IFN$\alpha 2,95 \mathrm{bp}$ in IFN- $\alpha 5,103 \mathrm{bp}$ in IFN- $\alpha 6,91 \mathrm{bp}$ in IFN- $\alpha 7$, and $165 \mathrm{bp}$ in IFN- $\beta$.

\section{qScript cDNA Synthesis}

cDNA was synthesized from mouse lung RNA to be used for RNA analysis with qScript cDNA synthesis kit. $5 \mathrm{uL}$ of nuclease water, $4 \mathrm{uL}$ of qScript Reaction Mix, $1 \mathrm{uL}$ of qScript Reverse Transcriptase, and 10uL of 100ng/uL sample RNA were added to each $0.1 \mathrm{~mL}$ tube in a 96-well PCR plate. Tubes were placed in a thermal cycler programmed as follows:

1 cycle: $22^{\circ} \mathrm{C}$ for 5 minutes

1 cycle: $42^{\circ} \mathrm{C}$ for 30 minutes

1 cycle: $85^{\circ} \mathrm{C}$ for 5 minutes

Upon the completion of the PCR cycle, cDNA was diluted at a 1:20 ratio with $342 \mathrm{uL}$ of TrisEDTA buffer. $360 \mathrm{uL}$ of cDNA was then stored in $-20^{\circ} \mathrm{C}$ refrigerator.

\section{Quantitative Real time PCR}

After primers were optimized, the primer solution that had the lowest cycle threshold (CT value), indicating the most amplification, was selected for quantitative real time PCR(qRT-PCR) on mice lungs samples. Each well contained $4 \mathrm{uL}$ of the selected primer (forward and reverse), $2 \mathrm{uL}$ of the sample's cDNA, 4 uL of nuclease free water, and 10uL of SYBR Green Master Mix. In addition to IFN primer, GAPDH primer, a housekeeping gene expressed constitutively in all mice, was also used at a concentration of 10 on each mouse's cDNA as positive controls of the experiment. qRT-PCR was carried out in a 96-Well 0.1-mL Block by QuantStudio® 5 System.

\section{$\Delta \triangle C T$ Calculations}

$\triangle \Delta \mathrm{CT}$ Calculation was done to analyze qRT-PCR data. The difference between IFN CT mean and CT mean of the positive control gene GAPDH $(\Delta \mathrm{CT})$ was calculated. $\Delta \mathrm{CT}$ mean of post-infection day 1 adult mice biological replicates was used as a reference for the following $\Delta \Delta \mathrm{CT}$ calculation for all samples. With $\Delta \Delta \mathrm{CT}$ values, I calculated percent expression using $100 * 2^{\wedge}(-\Delta \Delta \mathrm{CT})$ formula and standard deviation of the two biological replicates. Finally, T test was performed on adult and neonatal mice of the same day to evaluate the statistical significance of the data. 


\section{Results}

\section{Primer optimization}
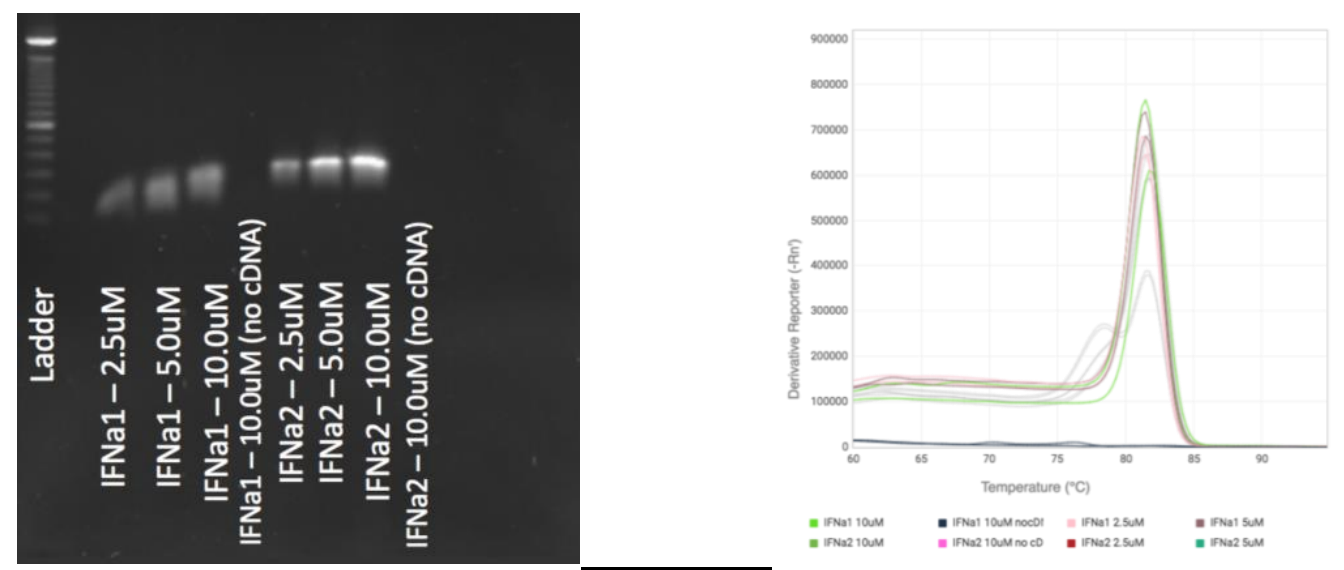

Figure 2. IFN $\alpha 1$, IFN $\alpha 2$ Gel Electrophoresis.

Figure 3. IFN- $\alpha 1$ Primer Optimization result.

Melt curve shows that IFN- $\alpha 1$ of concentration $10.0 \mu \mathrm{M}$ and $5.0 \mu \mathrm{M}$ have the highest peak, corresponding to largest amount of amplification.

In preparation for analysis of the lung samples primer pairs were optimized. IFN- $\alpha 1$ primer optimization result showed that, among all three concentrations, melt curves of $5.0 \mu \mathrm{M}$ and $10.0 \mu \mathrm{M}$ displayed the highest peaks. These two concentrations also had the lowest CT value. The negative control for IFN- $\alpha 1$ primer had no peak on its melt curve or amplification graph (Figure 3). The result from gel electrophoresis aligned with the melt curve, presenting one sole band of at roughly $127 \mathrm{bp}$ according to DNA ladder in its sample lanes (Figure 2).

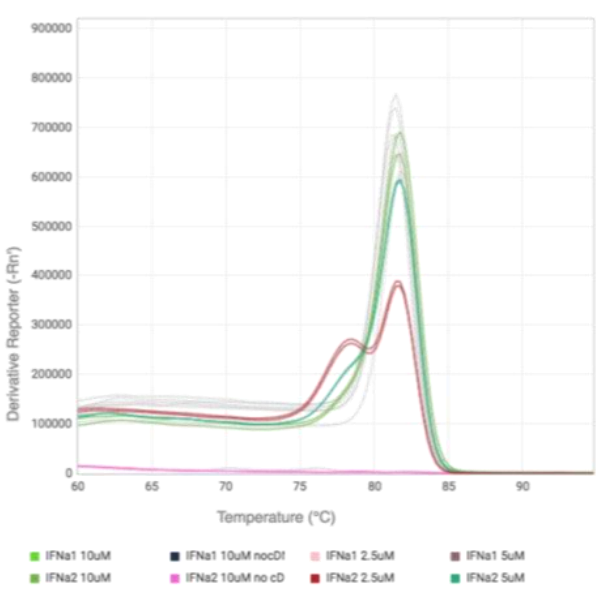

showing only one band of the correct size (Figure 2).
Figure 4. IFN- $\alpha 2$ Primer Optimization result.

Melt curve showed that IFN- $\alpha 2$ of concentration 10.0 $\mu \mathrm{M}$ and 5.0 $\mu \mathrm{M}$ had the highest peak, corresponding to largest amount of amplification. The flat line in negative control marked the absence of contamination in samples. Nevertheless, the double-peak in 2.5 concentration was a sign of the occurrence of primer dimer during amplification.

Similar to IFN- $\alpha 1$, IFN- $\alpha 2$ primer also had higher peaks in $5.0 \mu \mathrm{M}$ and $10.0 \mu \mathrm{M}$ melt curves. $5.0 \mu \mathrm{M}$ was determined the optimal concentration for RNA analysis despite the peak being slightly higher in $10.0 \mu \mathrm{M}$ primer, because of the insignificance of this minor difference (Figure 4). Gel electrophoresis for IFN- $\alpha 2$ confirmed this concentration selection by 


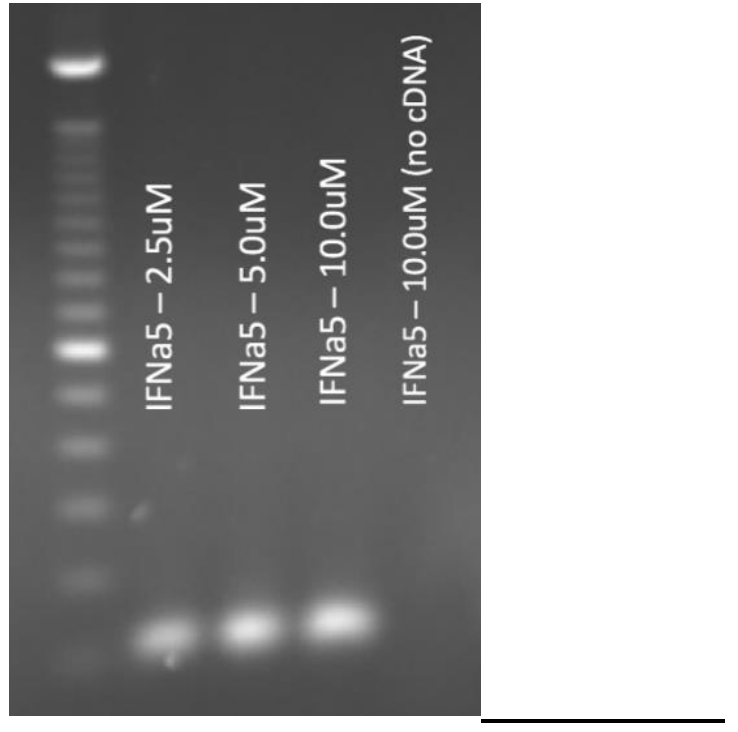

Figure 5. IFN- $\alpha 5$ gel electrophoresis.

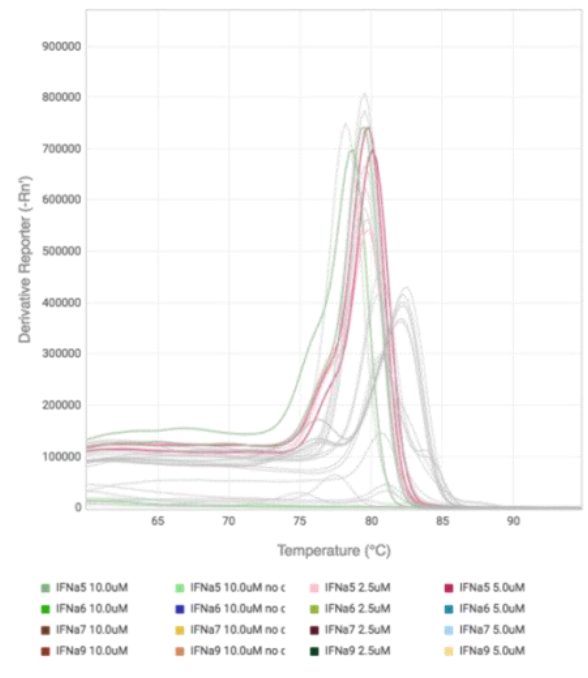

Figure 6. IFN- $\alpha 5$ Primer Optimization Result.

Melt curve showed that IFN- $\alpha 5$ of concentration $10.0 \mu \mathrm{M}$ and $5.0 \mu \mathrm{M}$ had the highest peak, corresponding to largest amount of amplification. Peaks in $10.0 \mu \mathrm{M}$ and $5.0 \mu \mathrm{M}$ melt curves were equally high (Figure 6). Thus, 5.0 $\mu \mathrm{M}$ IFN- $\alpha 5$ primer was selected for qPCR. A band of approximately $95 \mathrm{bp}$ was also seen in each IFN- $\alpha 5$ sample lanes on gel electrophoresis (Figure 5)

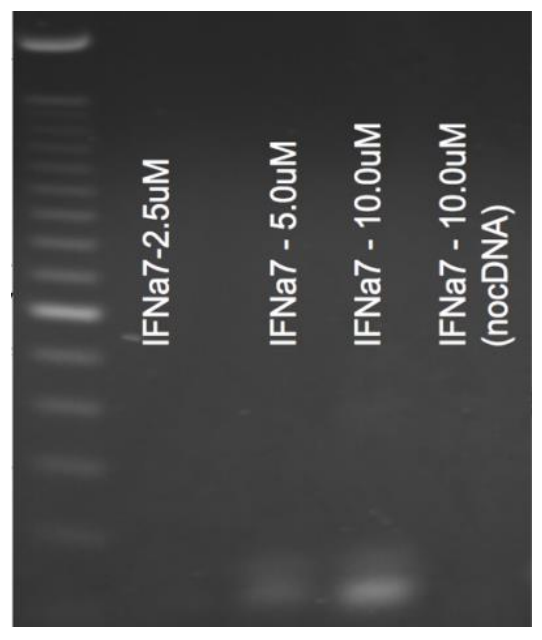

Figure 8. IFNa7 gel electrophoresis.

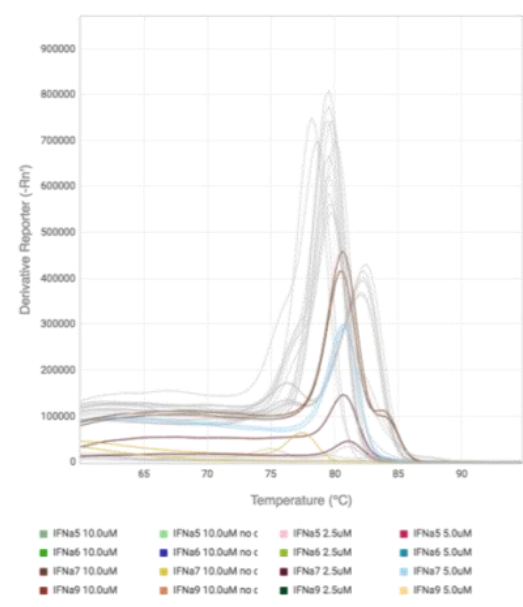

Figure 9. IFN- $\alpha 7$ primer Optimization result.

Melt curve showed that all concentrations of IFN- $\alpha 7$ primers yielded fewer amplicons than other interferon primers. $10.0 \mu \mathrm{M}$ had the highest peak. $10.0 \mu \mathrm{M}$ IFN- $\alpha 7$ displayed a much higher peak than that of others, so it was determined the best one for qPCR notwithstanding its weaker capability to amplify cDNA in comparison to other forms of IFN- $\alpha$ (Figure 9). 


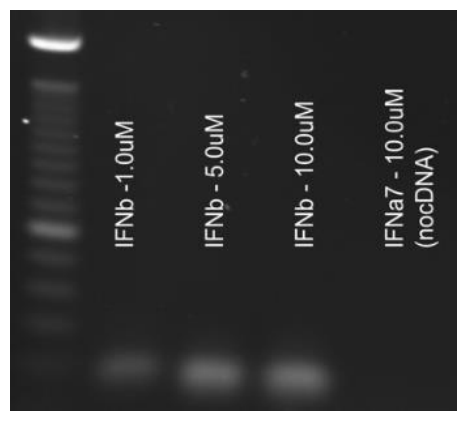

Figure 10. IFN- $\beta$ gel electrophoresis.
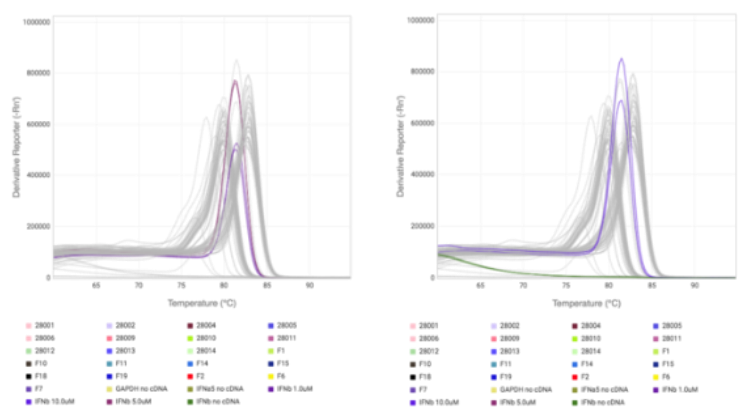

Figure 11. IFN- $\beta$ Primer Optimization Result.

Peaks in melt curves of $5.0 \mu \mathrm{M}$ and $10 \mu \mathrm{M}$ IFN- $\beta$ had virtually the same height. $5.0 \mu \mathrm{M}$ concentration was observed as the optimal because the two technical replicates perfectly overlapped and were not lower than $10.0 \mu \mathrm{M}$ by a large gap (Figure 11).

Type I IFN expression in adult and neonatal lungs

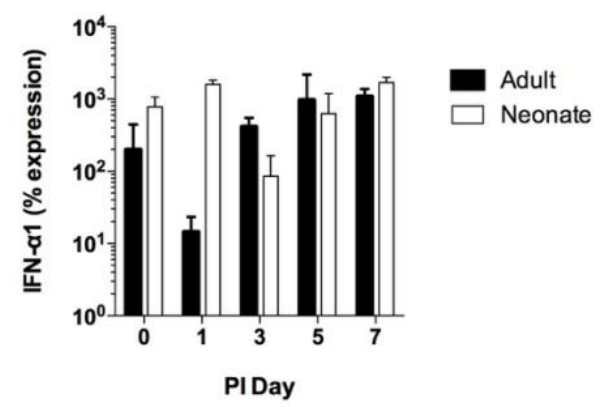

Figure 12. Adult (6-8 week old) and neonatal (2-day old) C57BL/6 mice were infected with $\mathrm{SeV}$ (500 pfu/g body weight) intranasally. Lungs were harvested after euthanasia on different days post-infection. IFN- $\alpha 1$ mRNA expression was determined by real-time PCR.

qRT-PCR result for IFN- $\alpha 1$ was consistent with the trend demonstrated in IFN- $\beta$ expression as previously shown - the expression in adult dropped from day 0 to day 1 , but increased daily following the decline. In contrast, its expression in neonates remained rather stable. There was no significant difference between adult in neonates in most days post-infection except for Day 1. The gap in expression level was greatest on Day 1.

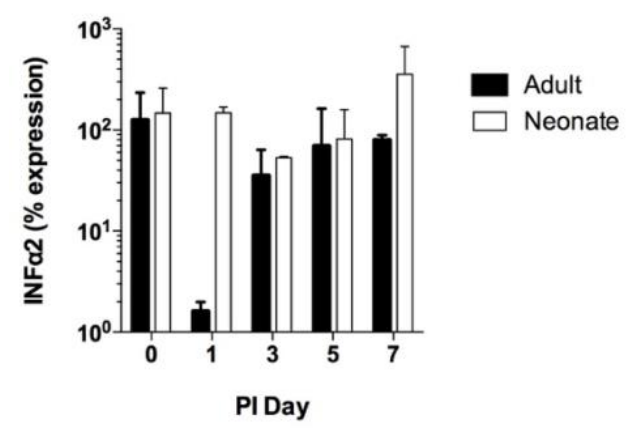

Figure 13. Adult (6-8 week old) and neonatal (2-day old) C57BL/6 mice were infected with $\mathrm{SeV}$ (500 pfu/g body weight) intranasally. Lungs were harvested after euthanasia on different days post-infection. IFN- $\alpha 2$ mRNA expression was determined by real-time PCR.

qRT-PCR for IFN- $\alpha 2$ showed a greater expression in neonatal mice in comparison to adult mice. This distinction was especially obvious on Day 1 . The trends in both age groups matched the trends in IFN- $\alpha 1$ expression. 


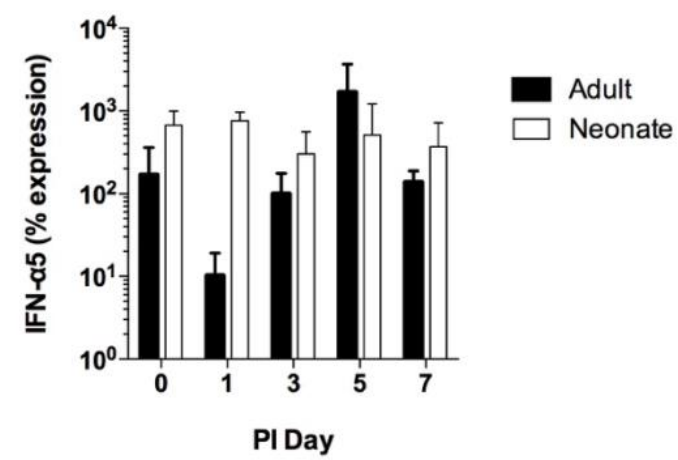

Figure 14. Adult (6-8 week old) and neonatal (2-day old) C57BL/6 mice were infected with $\mathrm{SeV}$ (500 pfu/g body weight) intranasally. Lungs were harvested after euthanasia on different days post-infection. IFN- $\alpha 5$ mRNA expression was determined by real-time PCR.

qRT-PCR result for IFN- $\alpha 5$ showed a similar trend in both age classes as compared to other interferons for the first five days. However, both exhibited downregulation on day 7 unlike other interferons.

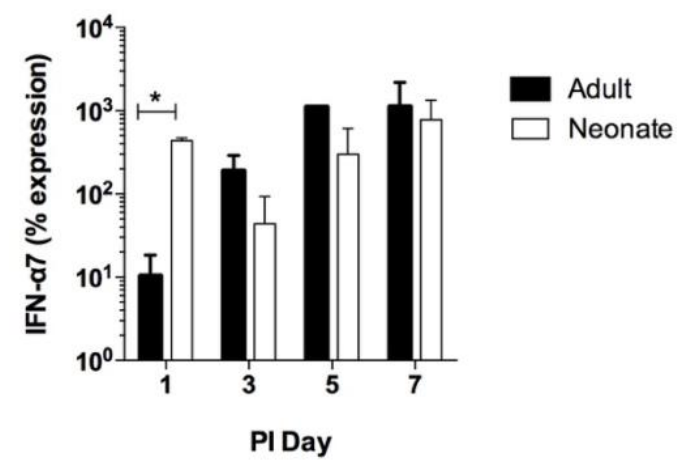

Figure 15. Adult (6-8 week old) and neonatal (2-day old) C57BL/6 mice were infected with $\mathrm{SeV}$ (500 pfu/g body weight) intranasally. Lungs were harvested after euthanasia on different days post-infection. IFN- $\alpha 7$ mRNA expression was determined bv real-time PCR.

qRT-PCR for IFN- $\alpha 7$ result showed that adult level was the lowest on day 1. Although neonatal level was significantly higher on day 1, it was reduced to be lower than adult level in since day 3 . Nonetheless, adult level increased over the course of infection and leveled off after day 5.

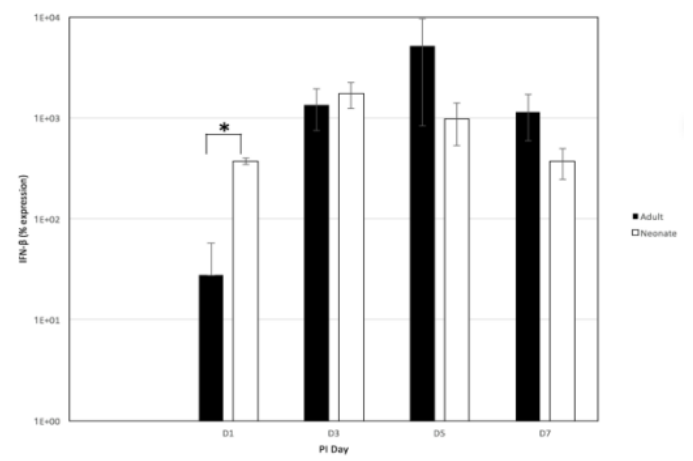

Figure 16. Adult (6-8 week old) and neonatal (2-day old) C57BL/6 mice were infected with $\mathrm{SeV}$ (500 pfu/g body weight) intranasally. Lungs were harvested after euthanasia on different days post-infection. IFN- $\beta$ mRNA expression was determined by real-time PCR.

Similar to IFN- $\alpha$, qRT-PCR showed that IFN- $\beta$ adult levels gradually increased from day 1 to day 5. It decreased back to day 3 level after day 5 as the virus was cleared by immune system. Especially, the difference between adult and neonatal expression was proven to be statistically significant by $\mathrm{T}$ test. In comparison, neonatal level remained stable, specifically, higher than adult level during early-infection stage. 


\section{Discussion}

Previous studies showed that IFN- $\beta$ levels were different in adult and neonatal mice during $\mathrm{SeV}$ infection. In adult lungs, IFN- $\beta$ mRNA levels increased over the course of infection but it was significantly reduced in neonatal lungs. In contrast, neonates had higher levels of IFN- $\beta$ protein.

It was interesting to note, that each of the IFN- $\alpha$ genes tested had higher baseline expression prior to viral infection, and this decreased by 24 hours post-infection followed by an increase immediately after infection. The neonates did not show a dip in expression at 24 hours following infection. Although the baseline differences on day 0 were not significant in this experiment, they may become significant with a larger group size.

Going forward, it is crucial to evaluate the protein amount of each of these interferons and see if the difference in IFN- $\beta$ holds true for all. Besides, interferons may not be the sole cause of this immune response variation, so more research has to be done on other types of cytokines.

\section{Conclusions}

The results showed that adult levels of IFN- $\alpha$ increased from day 1 to day 5 . In contrast, the neonatal levels were already increased at 24 hours post-infection. And these levels remained high. Statistically, there was significant differences between adult and neonatal lungs during the course of infection for IFN- $\alpha 7$ and IFN- $\beta$, which supported our hypothesis.

This experiment's hypothesis was not entirely supported due to the downregulation of all interferons on post-infection day 1 . The high expression on day 0 suggests that interferons reacted immediately to the infections. It is possible that other cytokines replaced the role often played by interferons on the first day in adults.

\section{Literature Citations}

Owen, Judith A., et al. Innate Immunity. 7th ed., New York, W.H. Freeman, 2013. 20 vols.

Bhattacharya et al.: Reduced inflammation and altered innate response in neonates during paramyxoviral infection. Virology Journal 2011 8:549.

McNab, Finlay, et al. "Type I interferons in infectious disease." Nature Reviews Immunology 15.2 (2015): 87.

Decker, Thomas, Mathias Müller, and Silvia Stockinger. "The yin and yang of type I interferon activity in bacterial infection." Nature Reviews Immunology 5.9 (2005): 675.

Noton, Sarah L., and Rachel Fearns. "Initiation and Regulation of Paramyxovirus Transcription and Replication." Virology 0 (2015): 545-554. PMC. Web. 6 July 2018.

Elias A. Said, Nicolas Tremblay, Mohammed S. Al-Balushi, Ali A. Al-Jabri, and Daniel Lamarre, "Viruses Seen by Our Cells: The Role of Viral RNA Sensors," Journal of Immunology Research, vol. 2018, Article ID 9480497, 14 pages, 2018. https://doi.org/10.1155/2018/9480497. 